\title{
Seguridad y eficacia del modelo de atención fast-track vs. atención convencional en apendicitis no complicada del paciente pediátrico
}

\author{
Safety and effectiveness of the fast-track attention model vs. conventional care in \\ uncomplicated appendicitis of the pediatric patient
}

\author{
Alberto Tlacuilo-Parra ${ }^{*}$, Sandy P. López-Valenzuela², Gabriela Ambriz-González² y \\ Elizabeth Guevara-Gutiérrez ${ }^{3}$ \\ 'División de Investigación en Salud, Unidad Médica de Alta Especialidad (UMAE) Hospital de Pediatría, Centro Médico Nacional de Occidente, \\ Instituto Mexicano del Seguro Social (IMSS); ${ }^{2}$ Departamento de Cirugía Pediátrica, UMAE Hospital de Pediatría, Centro Médico Nacional de \\ Occidente, IMSS; ${ }^{3}$ Departamento de Dermatología, Instituto Dermatológico de Jalisco. Guadalajara, Jalisco, México
}

\section{Resumen}

Objetivo: Comparar la seguridad y la eficacia de la atención fast-track vs. atención convencional en apendicitis no complicada en un hospital pediátrico. Método: Ensayo clínico controlado, aleatorizado, con dos grupos de 30 pacientes cada uno: A) fast-track, apendicitis no complicada que cumplieron el programa; y B) atención convencional, apendicitis no complicada con atención habitual. Variables de seguridad y eficacia: proporción de complicaciones y tiempo de estancia hospitalaria. Resultados: Se incluyeron 60 pacientes, sin diferencia entre grupos: sexo masculino (53 vs. 60\%), edad (8 \pm 3 vs. $8 \pm 2$ años), tiempo de evolución (23 \pm 21 vs. $24 \pm 20$ horas), tiempo desde ingreso a urgencias hasta inicio de cirugía (6 \pm 4 vs. $8 \pm 6$ horas), y tipo de apendicitis edematosa (27 vs. 24\%) o supurada (73 vs. $76 \%$ ). La estancia hospitalaria promedio del grupo fast-track fue de $13 \pm 5$ vs. $72 \pm 40$ horas del grupo de atención convencional $(p=0.001)$. Hubo complicaciones en el 3 y el $6 \%$, respectivamente $(p=1.0)$. La estancia hospitalaria disminuyó 2.45 días por paciente con el protocolo fast-track, lo que representa un ahorro de \$6,731 pesos/día/paciente/hospitalización (US\$373), sin un aumento de las complicaciones. Conclusión: El protocolo fast-track en los niños con apendicitis no complicada es seguro y efectivo en un hospital universitario. El programa fast-track aportó beneficios clínicos y económicos, ahorrando en total \$403,860 en los 30 pacientes.

PALABRAS CLAVE: Apendicitis. Apendicectomía. Cirugía fast-track. Análisis de costos. Ensayo clínico. Niños. Grupos relacionados por el diagnóstico.

\begin{abstract}
Objective: To compare safety and efficacy of fast-track program vs. conventional attention in non-complicated appendicitis attending a pediatric university hospital. Method: Randomized clinical trial, comparing two groups with 30 patients each: (A) fast-track group, appendicitis agreeing the treatment protocol; and (B) conventional attention group, appendicitis following habitual surgical care. The efficacy and safety measures were length of hospital stay and proportion of complications. Results: We included 60 patients, there were no significant difference between groups with regard: male gender (53 vs. $60 \%)$, age (8 \pm 3 vs. $8 \pm 2$ years-old), time of evolution (23 \pm 21 vs. $24 \pm 20$ h), time since admittance to emergency and beginning of surgery ( $6 \pm 4$ vs. $8 \pm 6$ h), and type of appendicitis edematous (27 vs. $24 \%$ ) or suppurate (73 vs. $76 \%$ ). Mean length of hospital stay in fast-track group was $13 \pm 5$ vs. $72 \pm 40 \mathrm{~h}$ in conventional attention $(p=0.001)$. The complications were 3 and
\end{abstract}

\author{
Correspondencia: \\ *Alberto Tlacuilo-Parra \\ Monte Olimpo 1413 \\ Col. Independencia \\ C.P. 44340 , Guadalajara, Jal., México \\ E-mail: albtlacuilo@yahoo.com
}

Fecha de recepción: 25-02-2018

Fecha de aceptación: 25-04-2018

DOI: 10.24875/CIRU.18000189
Cir Cir. 2018;86:412-416

Contents available at PubMed www.cirugiaycirujanos.com 
$6 \%$, respectively $(p=1.0)$. Fast-track program diminished length of hospital stay in 2.45 days per patient, representing a mean cost saving of 6,731 Mexican pesos per day, per patient hospitalized (US\$373), without increased complications. Conclusion: Fast-track program in children with non-complicated appendicitis is safe and effective in pediatric university hospital; there was cost-minimization without carelessness of safety. This program support clinical and economic benefits, a total saving of 403,860 Mexican pesos for the 30 patients in the fast-track group.

KEY WORDS: Appendicitis. Appendectomy. Fast-track surgery. Cost analysis. Clinical trial. Children. Diagnosis-related group.

\section{Introducción}

La apendicitis aguda es la enfermedad quirúrgica más frecuente en los servicios de cirugía y ocupa el primer lugar de las intervenciones quirúrgicas de abdomen agudo que se realizan en estos servicios en todo el mundo'.

La cirugía fast-track fue establecida por el cirujano danés Henrik Kehlet con la finalidad de optimizar el cuidado perioperatorio en la cirugía electiva ${ }^{2,3}$. El cuidado fast-track comprende un programa integral para reducir el estrés y el disconfort mediante instrucciones preoperatorias, movilización y alimentación posoperatoria inmediata, preferencia por técnicas mínimamente invasivas, así como tratamiento del dolor, evitando por otra parte drenes, tubos y catéteres ${ }^{4}$. Los estudios iniciales de cirugía fast-track en pediatría se han enfocado en procedimientos electivos tales como apendicectomía ${ }^{5,6}$, pielotomía y pieloplastía ${ }^{7,8}$, y nefrectomía ${ }^{9,10}$.

Según la evidencia sobre la factibilidad de implementar el modelo de atención fast-track aun en instituciones sin experiencia previa con este ${ }^{11}$, y dado que en nuestro medio el $47 \%$ de las cirugías de apendicitis corresponden a formas no complicadas (edematosa y supurada) ${ }^{12}$, consideramos útil comparar la seguridad y la eficacia del protocolo de atención fasttrack vs. atención convencional en niños con apendicitis no complicada en un hospital universitario pediátrico.

\section{Método}

Ensayo clínico aleatorizado, controlado, efectuado de julio a diciembre de 2015 en la Unidad Médica de Alta Especialidad (UMAE) Hospital de Pediatría del Centro Médico Nacional de Occidente del Instituto Mexicano del Seguro Social (IMSS). Se calculó una muestra basada en obtener una diferencia de al menos 48 horas entre las medias de estancia hospitalaria entre el grupo fast-track y el de atención convencional, con un total de 30 sujetos de estudio por grupo, asignados mediante aleatorización simple utilizando una tabla de números. Las variables estudiadas fueron las siguientes: edad, sexo, turno de ingreso, referencia al hospital, tiempo de evolución, tiempo transcurrido al momento de iniciar la cirugía, tiempo de estancia hospitalaria, complicaciones quirúrgicas, condiciones comórbidas asociadas y motivo de egreso. Se incluyeron pacientes entre 3 y 15 años de edad atendidos en el servicio de urgencias con diagnóstico clínico de apendicitis no complicada, corroborada durante el acto quirúrgico y operada mediante apendicectomía abierta.

\section{Protocolo fast-track y atención convencional}

Se aplicó una cédula para registrar las características demográficas, cuantificando el tiempo transcurrido desde el ingreso al hospital hasta el momento de la cirugía. Se aplicó un esquema antibiótico profiláctico en el momento de la inducción anestésica: cefazolina (30 mg/kg por vía intravenosa en dosis única) más metronidazol (30 mg/kg por 5 días); en caso de hipersensibilidad a las cefalosporinas se utilizó amikacina ( $15 \mathrm{mg} / \mathrm{kg}$ por 3 días). Al momento de egreso del quirófano se entrevistó al padre o tutor, se le explicó el objetivo del estudio y posterior a la firma del consentimiento bajo información se aleatorizó para pertenecer al protocolo de atención fast-track: analgesia con paracetamol $(15 \mathrm{mg} / \mathrm{kg}$ por vía oral cada 6 horas, máximo $3 \mathrm{~g} /$ día) por 3 días, movilización temprana fuera de la cama y alimentación posoperatoria temprana (a las 6 horas del posquirúrgico se inició la vía oral de acuerdo con los siguientes parámetros: sin presencia de vómito, sin distensión ni dolor abdominal, con presencia de peristalsis, con canalización de gases o presencia de evacuaciones); o para recibir atención convencional (según el juicio clínico del cirujano pediatra responsable de la atención del paciente). Se realizaron las indicaciones correspondientes según el protocolo de atención asignado. Se cuantificaron las horas de estancia hospitalaria desde el inicio de la cirugía hasta el egreso, si se desarrolló alguna complicación intrahospitalaria y las razones de egreso hospitalario. Dentro del 
protocolo fast-track, los pacientes debía contar con los siguientes criterios de egreso: afebril, presencia de ruidos peristálticos o canalizando gases, tolerancia a líquidos, y sin náuseas ni vómitos. A los 7 días de efectuada la cirugía se realizó llamada telefónica a ambos grupos para determinar si hubo complicaciones (reingreso hospitalario o reintervención).

\section{Grupos relacionados por el diagnóstico}

Los grupos relacionados por el diagnóstico (GRD) son un sistema de clasificación y agrupación de los diagnósticos clínicos y quirúrgicos de los pacientes hospitalizados, y definen el nivel de complejidad que involucra la atención de un paciente. Cada uno de los GRD se construye de acuerdo con los diagnósticos de egreso, combinando las diversas características clínicas (clasificadas mediante las claves CIE-10) y los procedimientos quirúrgicos realizados (claves CIE$9 \mathrm{MC}$ ), e incluyen de manera predominante el consumo de recursos. La apendicectomía fue clasificada, en función de los GRD-IMSS, dentro de seis categorías existentes: GRD 338, apendicectomía con diagnóstico principal complicado con complicaciones clínicas mayores; GRD 339, apendicectomía con diagnóstico principal complicado con complicaciones clínicas; GRD 340, apendicectomía con diagnóstico principal complicado sin complicaciones clínicas ni complicaciones clínicas mayores; GRD 341, apendicectomía sin diagnóstico principal complicado con complicaciones clínicas mayores; GRD 342, apendicectomía sin diagnóstico principal complicado con complicaciones clínicas; y GRD 343, apendicectomía sin diagnóstico principal complicado sin complicaciones clínicas ni complicaciones clínicas mayores ${ }^{13}$.

\section{Análisis estadísticos}

Se utilizó estadística descriptiva: promedios, desviaciones estándar y proporciones. Para la estadística inferencial se usó la prueba de $\chi^{2}$, o la prueba exacta de Fisher para comparar proporciones, y la prueba $t$ de Student para variables cuantitativas entre grupos. Se consideró una diferencia estadísticamente significativa cuando $p<0.05$. Se realizó una evaluación económica de tipo costo-minimización para ambos modelos de atención con respecto a las horas de estancia hospitalaria real; este análisis asume la evaluación de al menos dos estrategias en una misma enfermedad en las que las respuestas obtenidas en salud son iguales, pero el costo es diferente. Para calcular el costo de la estancia hospitalaria se empleó el tabulador de costos unitarios por nivel de atención médica para el ejercicio 2015 del IMSS, publicado en el Diario Oficial de la Federación ${ }^{14}$, que establece que el costo del día/paciente en hospitalización en unidades de tercer nivel de atención es de \$ 6.731. Este trabajo contó con la aprobación del Comité Local de Investigación y Ética en Investigación (R-2015-130234) de la UMAE Hospital de Pediatría.

\section{Resultados}

\section{Características sociodemográficas del grupo de atención fast-track}

Se incluyeron 30 pacientes pediátricos con diagnóstico de apendicitis no complicada, atendidos durante un periodo de 6 meses. Los datos demográficos comparativos entre grupos se muestran en la tabla 1. La distribución del tipo de apendicitis clasificada al momento de la cirugía fue edematosa en 8 casos (27\%) y supurada en 22 casos $(73 \%)$, la cual fue corroborada mediante estudio histopatológico. Con respecto al empleo de antibiótico, este se aplicó de manera preoperatoria en 28 casos (93\%), mientras que en los dos restantes se administró de manera intraoperatoria (7\%). La estancia hospitalaria promedio fue de $13 \pm 5$ horas. Se presentaron complicaciones quirúrgicas en un caso (3\%), que correspondió a un seroma que se resolvió mediante aspiración. Ninguno de los pacientes presentó ninguna condición comórbida asociada. Los 30 pacientes (100\%) fueron dados de alta por mejoría, y durante la llamada de seguimiento a los 7 días del alta hospitalaria, para confirmar la seguridad del paciente, en ningún caso se refirió la necesidad de reingreso hospitalario y se corroboró el regreso a las actividades cotidianas de los pacientes.

\section{Características sociodemográficas del grupo de atención convencional}

Se incluyeron 30 pacientes con diagnóstico de apendicitis no complicada. La distribución del tipo de apendicitis clasificada al momento de la cirugía fue edematosa en 7 casos (24\%) y supurada en 23 casos (76\%). Con respecto al empleo de antibiótico, este se aplicó de manera preoperatoria en 29 casos (96\%), mientras que en el restante se administró de manera intraoperatoria (4\%). La estancia hospitalaria promedio fue de $72 \pm 40$ horas. Se presentaron complicaciones quirúrgicas en dos casos (6\%), que correspondieron a seromas que se resolvieron mediante aspiración. Ninguno 
Tabla 1. Comparación entre el modelo de atención fast-track y la atención convencional

\begin{tabular}{|c|c|c|c|}
\hline & \multicolumn{2}{|c|}{$(\mathrm{N}=30)$} & \multirow[t]{2}{*}{ p } \\
\hline & $\begin{array}{l}\text { Atención } \\
\text { fast-track }\end{array}$ & $\begin{array}{c}\text { Atención } \\
\text { convencional }\end{array}$ & \\
\hline Edad (años) & $8 \pm 3$ & $8 \pm 2$ & 0.840 \\
\hline Sexo masculino & $16(53 \%)$ & $18(60 \%)$ & 0.602 \\
\hline $\begin{array}{l}\text { Turno de ingreso } \\
\text { Matutino } \\
\text { Vespertino } \\
\text { Nocturno } \\
\text { Jornada acumulada }\end{array}$ & $\begin{array}{c}2(7 \%) \\
4(13 \%) \\
16(53 \%) \\
8(27 \%)\end{array}$ & $\begin{array}{c}1(3 \%) \\
3(10 \%) \\
18(60 \%) \\
8(27 \%)\end{array}$ & 0.897 \\
\hline Referido al hospital & $26(87 \%)$ & $27(90 \%)$ & 1.00 \\
\hline $\begin{array}{l}\text { Modalidad diagnóstica } \\
\text { radiografía simple }\end{array}$ & $27(90 \%)$ & $27(90 \%)$ & 1.00 \\
\hline $\begin{array}{l}\text { Tiempo de } \\
\text { evolución (horas) }\end{array}$ & $23 \pm 21$ & $24 \pm 20$ & 0.71 \\
\hline $\begin{array}{l}\text { Tiempo al inicio de } \\
\text { cirugía (horas) }\end{array}$ & $6 \pm 4$ & $8 \pm 6$ & 0.104 \\
\hline $\begin{array}{l}\text { Estancia } \\
\text { hospitalaria (horas) }\end{array}$ & $13 \pm 5$ & $72 \pm 40$ & 0.0001 \\
\hline $\begin{array}{l}\text { Complicaciones } \\
\text { quirúrgicas }\end{array}$ & $1(3 \%)$ & $2(6 \%)$ & 1.0 \\
\hline Condiciones comórbidas & 0 & 0 & 1.0 \\
\hline Motivo de egreso mejoría & $30(100 \%)$ & $30(100 \%)$ & 1.0 \\
\hline
\end{tabular}

de los pacientes presentó ninguna condición comórbida asociada. El $100 \%$ de los niños fueron dados de alta por mejoría, y durante la llamada de seguimiento a los 7 días del alta hospitalaria en ningún caso se refirió la necesidad de reingreso hospitalario y se confirmó el regreso a las actividades cotidianas.

\section{Comparación entre modelos de atención}

No hubo diferencia estadísticamente significativa en cuanto a la clasificación de la apendicitis entre grupos. La mayoría de los pacientes fueron referidos de otras unidades hospitalarias, y el $50 \%$ ingresaron durante el turno nocturno. La modalidad de imagen más útil para establecer el diagnóstico fue la radiografía simple de abdomen, en el $90 \%$ de los casos. No hubo diferencia entre grupos respecto al tiempo de evolución promedio desde el inicio de la sintomatología hasta que fueron ingresados a alguna unidad hospitalaria, ni en el tiempo transcurrido en horas desde su ingreso a nuestra unidad y el inicio de la cirugía. El tiempo promedio de estancia hospitalaria en el grupo fast-track fue de $13 \pm$ 5 horas, y en el grupo de atención convencional fue de
$72 \pm 40$ horas $(p=0.0001)$. No existió diferencia en la proporción de complicaciones quirúrgicas, reingresos o condiciones comórbidas entre los modelos de atención. Todos los pacientes fueron egresados por mejoría y todos se integraron a sus actividades cotidianas sin desarrollar secuelas.

\section{Clasificación de la apendicitis mediante GRD y costo-minimización}

El GRD 343, apendicectomía sin diagnóstico principal complicado, sin complicaciones mayores ni comorbilidad, comprendió 29 pacientes $(97 \%)$ del grupo fast-track y 28 (94\%) del modelo de atención convencional. El GRD 342, apendicectomía sin diagnóstico principal complicado, con complicaciones clínicas, correspondió a 1 paciente (3\%) y 2 pacientes $(6 \%)$, respectivamente.

En el grupo fast-track, la estancia hospitalaria promedio fue de $13 \pm 5$ horas vs. $72 \pm 40$ horas en el modelo de atención convencional, con una diferencia entre grupos de 59 horas (2.45 días), lo cual traduce una reducción de los costos de por lo menos \$13,462 M.N. (considerando solo los 2 días y eliminando la fracción de horas restantes) por paciente atendido de apendicectomía abierta bajo el modelo de atención fast-track.

\section{Discusión}

La aplicación del modelo fast-track a las intervenciones quirúrgicas urgentes parece estar aportando buenos resultados en aquellas patologías que no presenten complicaciones graves. En estos casos, la actuación sobre la fase preoperatoria se hace más difícil, ya que el tiempo entre el diagnóstico y la intervención quirúrgica abarca apenas unas horas, y la situación clínica del paciente hace que, en la mayoría de los casos, el control nutricio y metabólico no sea el adecuado ${ }^{15}$. Así, toman más relevancia las etapas intraoperatoria y posoperatoria. No obstante, previo a la intervención quirúrgica urgente se debe iniciar el protocolo de información y educación, además de la profilaxis antibiótica y la reanimación hidroelectrolítica, intentando que el paciente llegue a la cirugía con una volemia adecuada que asegure la perfusión de los tejidos sin edematizarlos ${ }^{16,17}$.

La apendicitis aguda es la patología quirúrgica urgente más frecuente en el niño, pero aún no existe consenso en cuanto a su manejo terapéutico. En la literatura existen varios estudios sobre el tratamiento 
mediante vías rápidas de la apendicitis aguda no complicada de la edad pediátrica. En ellos se logra una recuperación posoperatoria rápida, semejando los tiempos de los protocolos fast-track de las intervenciones quirúrgicas programadas. Dos piezas clave de su éxito son la utilización de la vía laparoscópica como técnica quirúrgica, sobre todo en casos seleccionados (niñas en edad puberal y pacientes obesos), y la implantación de dosis antibiótica como profilaxis ${ }^{18,19}$. Otro punto cardinal en el modelo de atención fast-track es evitar drenajes peritoneales para prevenir la formación de abscesos, así como evitar la colocación de una sonda nasogástrica para prevenir la distensión abdominal y los vómitos, pues hoy en día se afirma que estas dos últimas acciones no presentan ventajas frente a no llevarlas a cabo e incluso pueden aumentar la presencia de complicaciones ${ }^{20,21}$. La tolerancia oral precoz mejora el confort del paciente y disminuye la estancia hospitalaria ${ }^{22}$. A ello se suma la existencia de trabajos que respaldan la tolerancia precoz posoperatoria en los casos de apendicitis aguda complicada con el fin de disminuir el íleo paralítico ${ }^{15}$.

La aplicación de un modelo de atención fast-track en la apendicitis aguda no complicada en el escenario de un hospital universitario es factible y útil cuando se compara con el modelo de atención convencional, traduciéndose en beneficios clínicos: a) disminución de la estancia hospitalaria en 2.4 días, lo cual significa una reducción de la ansiedad y del estrés tanto para el paciente como para su familia y una pronta integración a la dinámica familiar habitual; b) calidad de la atención, ya que en la llamada que se realizó a los pacientes 7 días después de la intervención, con la finalidad de verificar la seguridad del procedimiento y evitar complicaciones o reingresos, se percibió como una mejora en la calidad de la atención brindada; y c) reducción en el riesgo de desarrollar infección nosocomial. Igualmente se traduce en beneficios económicos, que se ven reflejados en la disminución de la estancia hospitalaria en más de 2 días por paciente, lo que supone una reducción promedio de $\$ 13,462$ por paciente hospitalizado. Si consideramos que derivado de la publicación previa de nuestro grupo ${ }^{12}$ el $47 \%$ de los pacientes pediátricos con apendicitis presentan formas no complicadas, y que durante el año 2014 se realizaron en la unidad 386 apendicectomías, 185 de ellas corresponderían a formas no complicadas, lo cual representaría un ahorro total de $\$ 2,490,470$, sin menoscabo de la seguridad del paciente.

Nuestro estudio presenta limitaciones que debemos reconocer. El tamaño de la muestra es pequeño, dado que se trata de un estudio piloto cuyo objetivo era determinar la factibilidad de establecer un modelo de atención fast-track en el escenario de un hospital universitario. Adicionalmente, el protocolo terapéutico se redujo a la labor del cirujano y principalmente a su actuación en la fase posoperatoria, sin considerar el papel del anestesiólogo, por lo que el concepto del modelo fast-track es incompleto.

\section{Conflicto de intereses}

\section{Ninguno por declarar.}

\section{Bibliografía}

1. Humes DJ, Simpson J. Acute appendicitis. BMJ. 2006:335:530-4.

2. Kehlet $\mathrm{H}$. Effect of postoperative pain treatment and outcome-current status and future strategies. Langenbecks Arch Surg. 2004;389:244-9.

3. Wilmore DW, Kehlet $\mathrm{H}$. Management of patients in fast-track surgery. BMJ. 2001;322:473-6.

4. Reismann M, Arar M, Hofmann A, Schukfeh N, Ure B. Feasibility of fasttrack elements in pediatric surgery. Eur J Ped Surg. 2012;22:40-4.

5. Grewal H, Sweat J, Vázquez WD. Laparoscopic appendectomy in children can be done as fast-track or same day surgery. J Soc Laparoendosc Surg. 2004;8:151-4.

6. Serour F, Witzling M, Gorenstein A. Is laparoscopic appendectomy in children associated with an uncommon postoperative complication? Surg Endosc. 2005;19:919-22.

7. Mohamed M, Hollins G, Eissa M. Experience in performing pyelolithotomy and pyeloplasty in children on day surgery basis. Urology. 2004;64:1220-2.

8. Metzelder ML, Schier F, Petersen C. Laparoscopic trans-abdominal pyeloplasty in children is feasible irrespective of age. J Urol. 2006;175:688-91.

9. Mulholland TL, Kropp BP, Wong C. Laparoscopic renal surgery in infants $10 \mathrm{~kg}$ or less. J Endourol. 2005;19:397-400.

10. Jesch NK, Metzelder ML, Kuebler JF. Laparoscopic trans-peritoneal nephrectomy is feasible in the first year of life and is not afected by kidney size. J Urol. 2006;176:1177-9.

11. Schukfeh N, Reismann M, Ludwikowski B, Hofmann AD, Metzelder ML, Ure B. Implementation of fast-track pediatric surgery in a German nonacademic institution without previous fast-track experience. Eur J Pediatr Surg. 2014;24:419-25

12. Tlacuilo-Parra A, Hernández-Hernández A, Venegas-Dávalos $M$, Gutiérrez-Hermosillo V, Guevara-Gutiérrez E, Ambriz-González G. Costos de tratamiento de la apendicitis mediante grupos relacionados con el diagnóstico en un tercer nivel de atención pediátrica. Cir Cir. 2014;82:628-36.

13. Arroyave LMG, Aburto MR, Dávila TJ, editores. Enfermedades y trastornos del sistema digestivo. En: Grupos relacionados con el diagnóstico: producto hospitalario. GRD-IMSS: 2012. México, DF: Instituto Mexicano del Seguro Social; 2014. p. 57-70.

14. Diario Oficial de la Federación. Acuerdo ACDO.AS3.HCT.280115/7.P.DF y sus Anexos, dictado por el $\mathrm{H}$. Consejo Técnico, celebrado el 28 de enero de 2015, relativo a la aprobación de los Costos Unitarios por Nivel de Atención Médica para el ejercicio 2015 del IMSS. Diario Oficial, miércoles 11 de febrero de 2015.

15. Lasso B, Ruiz H, Vargas V. Aplicación de un modelo terapéutico fasttrack en la apendicitis aguda complicada del paciente pediátrico. Cir Pediatr. 2013;26:63-8.

16. Holte $\mathrm{K}$, Kehlet $\mathrm{H}$. Fluid therapy and surgical outcomes in elective surgery: a need for reassessment in fast-track surgery. J Am Coll Surg. 2006;202:971-89.

17. Tuduri L, Morcillo A, Granero C. Protocolo antibiotico "fast-track" en la apendicitis aguda. Cir Pediatr. 2009;22:142-4.

18. Wojciech J, Gronchowski JA. Are antibiotics necessary in nonperforated appendicitis in children? A doble blind randomized controlled trial. Med Sci Monit. 2001;7:289-92.

19. Reissman M, Kampen M, Laupichler B. Fast-track surgery in infants and children. J Pediatr Surg. 2007;42:234-8.

20. Perovic Z. Drainage of the abdominal cavity and complications in perforating appendicitis in children. Med Pregl. 2000;53:193-6.

21. Jottard K, Hoff C, Maessen J. Life and death of nasogastric tube in elective colonic surgery in the Netherlands. Clin Nutr. 2009;28:26-8.

22. Chopra S, Schmidt SC, Fotopoulou C. Evidence-based perioperative management: strategic shitfs in times of fast track surgery. Anticancer Res. 2009;29:2799-802. 